\title{
Carnets
}

Revue électronique d'études françaises de l'APEF

Première Série - 4 Numéro Spécial | 2012

Invasions \& Évasions

\section{Rencontres précoloniales : conflits maritimes entre la France et le Portugal avant 1580}

\section{Géorges van den Abbeele}

\section{(2) OpenEdition}

Journals

Édition électronique

URL : http://journals.openedition.org/carnets/7547

DOI : 10.4000/carnets. 7547

ISSN : 1646-7698

Éditeur

APEF

Édition imprimée

Date de publication : 1 juin 2012

Pagination : 147-154

Référence électronique

Géorges van den Abbeele, «Rencontres précoloniales : conflits maritimes entre la France et le

Portugal avant 1580 », Carnets [En ligne], Première Série - 4 Numéro Spécial | 2012, mis en ligne le 23

juin 2018, consulté le 01 mai 2019. URL : http://journals.openedition.org/carnets/7547 ; DOI :

$10.4000 /$ carnets. 7547

\section{(c) (1) (8)}

Carnets est mis à disposition selon les termes de la licence Creative Commons - Atribution - Pas d'utilisation commerciale 4.0 International. 


\title{
RENCONTRES PRECOLONIALES. Conflits maritimes entre la France et le Portugal avant 1580
}

\author{
Georges Van Den AbBeele \\ Northeastern University, Boston \\ g.vandenabbeele@neu.edu
}

\section{Résumé}

Le sujet de cette communication traite des guerres et des conflits entre les Français et les Portugais en mer Atlantique aux XVe et XVle siècles. Depuis les luttes pour les Canaries et les Açores, et la concurrence entre les pêcheurs dans les grands bancs de Terre-Neuve et du Labrador, jusqu'aux grandes expéditions coloniales et sanglantes dans la baie de Guanabara, la France et le Portugal se sont disputés de vastes champs d'empire océanique pour les perdre tous les deux, grâce d'une part aux guerres de religion (la France) et d'autre part à la grande mésaventure marocaine (le Portugal) tandis que l'Espagne et l'Angleterre profitent de leurs déboires. Quelles sont les conséquences à long terme - économique, géopolitique, symbolique - de cette double défaite dans un dédale d"'invasions et évasions "?

\section{Abstract}

This paper addresses the wars and conflicts between the French and the Portuguese in the Atlantic Ocean during the fifteenth and sixteenth centuries. From the struggles over the Canary Islands and the Azores, and the competition for fishing in the Great Banks of Newfoundland and Labrador, up through the great, bloody colonial expeditions in Guanabara Bay, France and Portugal fought over the vast expanses of their sea empires only for both countries to lose their gains, thanks to the wars of religion in France, on the one hand, and the great misfortune that befell Portugal in Morocco, on the other hand. What are the long-term consequences - economically, geopolitically, and symbolically of this twin defeat amidst a labyrinth of "invasions and evasions"?

Mots-clés: Colonialisme, Nationalisme, Histoire navale, pêche de la morue Keywords: Colonialism, Nationalism, naval History, cod fishing. 
Bien avant que Napoléon ait conçu l'idée même d'envoyer ses troupes à travers l'Espagne en direction du Portugal, Français et Portugais se sont affrontés dans des rencontres armées que l'on ne traitera moins en termes d'invasions (ou d'évasions) qu'en termes d'incursions par où une France (qui se voit en quelque sorte comme le tiers exclu du Traité de Tordesillas, selon lequel le pape Alexandre VI en 1494 divise le monde entre la couronne de Castille et celle du Portugal), une France, bref, qui se sent exclue de la nouvelle réalité mondialisée issue précisément des découvertes ibériques, essaie de s'imposer d'une manière ou d'une autre dans le nouvel ordre, par force s'il le faut.

Or, cette lutte entre la France et le Portugal se voit jouer sur trois plans différents, depuis le début du $X V^{e}$ siècle jusqu'à la fin du $X V l^{e}$. A la fin de cette époque, et pour des raisons très différentes, ces deux pays se voient sombrer dans des catastrophes nationales qui auraient eu l'effet de démunir leurs espoirs impériaux à tel point que leurs grands rivaux - l'Espagne, la Hollande, et surtout l'Angleterre - en ont tiré des avantages définitifs dans leurs quêtes à eux de la domination mondiale, des avantages qui, il ne faut guère le dire, restent jusqu'à nos jours, et qui déterminent beaucoup de ce qu'on appelle à l'heure actuelle la "mondialisation", y compris la domination actuelle et mondiale des langues anglaises et hispaniques.

Ces deux catastrophes sont évidemment les conséquences affreuses de la terrible défaite du roi Sébastien sur les champs d'Alcácer-Quibir en 1578, et les suites non moins douloureuses et brutales des guerres de religion déclenchées par le massacre de la Saint Barthélemy en 1572.

Avant ces deux moments catastrophiques, Français et Portugais s'affrontaient sur les trois plans indiqués qui sont :1) les luttes pour les îles de l'Atlantique ; 2) la rivalité sur les grands bancs et l'accès à la pêche de la morue ; et 3 ) la guerre au Brésil.

Que l'on nous permettre donc d'analyser ces trois points de friction entre les deux pays et leurs conséquences parfois très lointaines.

Sur notre premier plan, commençons d'abord avec l'invasion des Canaries. En 1402, Jean IV de Béthencourt mène une armée de ses compatriotes normands et gascons qui, malgré leur pays d'origine, vouaient leurs services au roi de Castille, Henri III. Celui-ci, en récompense, ne trouva rien de mieux qu'à décerner au sieur de Béthencourt le titre de "roi des Canaries" avec la seule restriction que ce soi-disant roi lui vouât par retour son unique hommage vassalique au roi castillan. Malgré cet appui majeur, Béthencourt et compagnie ont eu le plus grand mal à dompter les indigènes canariens qui lui montaient une résistance acharnée et durable. N'empêche qu'une épopée française, Le Canarien, arrive à clamer les "exploits" du grand héros normand. Vingt ans plus tard, arrivent les Portugais avec une autre force armée qui ne réussit pas mieux que les Français. S'ensuit l'établissement d'une zone de conflit qui dure la plupart du XVe siècle jusqu'au Traité d'Alcáçovas en 1479, qui décida 
en faveur des prétentions castillanes pour les Canaries tout en obligeant l'Espagne à reconnaître les droits du Portugal sur les îles de Madère, des Açores, et du Cap Vert, aussi bien que "toutes autres îles, terres, et côtes découvertes ou à découvrir, trouvées ou à trouver" . Ce traité présuppose effectivement le désir ou l'angoisse du Portugal de sauvegarder ses prétentions sur Porto Santo, Madère et les Açores, tous découverts et peuplés depuis la troisième décennie du $\mathrm{XV}^{\mathrm{e}}$ siècle. Et effectivement, le Portugal s'est trouvé dans la nécessité de protéger ces territoires insulaires, mais moins contre l'Espagne que contre toute une suite d'incursions françaises depuis le corsaire Coulam en 1469, l'armateur breton Mondragon, le dieppois Jean Fleury, le corsaire Moluc et bien d'autres jusqu'au siège de Ponto Delgado par une flotte française dirigée par le condottiere italien, Filippo di Piero Strozzi en 1582, à la fin de l'époque qui nous regarde (Pereira, $2010: 114,151,183,198-99$, 218-20 ; Morison, 1971 : 254 ; Russell-Wood, 1992 : 73). Reste le bilan évident que sur ce plan de conflits franco-portugais, c'est bel et bien le Portugal qui a gagné et cela de manière bien définitive.

Reste le deuxième plan, dont les contours du conflit se dessinent déjà par la fin du $\mathrm{XV}^{\mathrm{e}}$ siècle pour s'éclater ouvertement au $\mathrm{XVI} \mathrm{e}^{\mathrm{e}}$. Or, si les faits restent essentiellement incontestés en ce qui concerne les découvertes portugaises des côtes de l'Afrique et du Brésil, d'une part, et d'autre part, celles des Antilles par Christophe Colomb au service du roi d'Espagne, la situation en Amérique du nord est moins claire. On ne fait plus de doute que les Vikings y ont bien voyagé dès le $\mathrm{XI}^{\mathrm{e}}$ siècle, mais c'est au Portugais Lavrador que va l'honneur d'avoir légué son nom à la côte nord-est de l'actuel Canada lors de son voyage dans les parages de Groenland en $1499^{2}$. Deux ans plus tôt, I'Italien Giovanni Caboto, au service du roi d'Angleterre Henri VII, avait côtoyé la Terre-Neuve, suivi trois ans plus tard, par les frères Gaspard et Miguel Corte-Real qui, eux, ont bien pris possession de ce pays au nom de roi du Portugal, comme témoigne surtout la carte planisphère dite de Cantino faite en 1502 et aujourd'hui conservée à la Biblioteca Estense à Modena. Malheureusement, les deux frères Corte-Real sont disparus et présumés morts dans leurs voyages subséquents sur la côte nord-américaine, qu'ils auraient visitée depuis la Floride jusqu'au Labrador (Lopes, 1991: 13-22) ${ }^{3}$. Jusqu'au milieu du XVII ${ }^{\mathrm{e}}$ siècle (voir, par exemple, le grand globe

\footnotetext{
${ }^{1}$ [y cualesquiera otras islas e tierras, costas descubiertas e por descubir, halladas e por hallar], "Traslado de los capítulo as del tratado de paces entre las coronas de Castilla y de Portugal firmado en Toledo á 16 de Marzo de 1480, relátivos á la posesión y pertenencia de Guinea, costas, mares é islas de África ", Boletin de la real Academia de la Historia, p. 327.

2 L'itinéraire de ce voyage reste néanmoins très disputé, depuis une simple méprise sur la côte du Groenland jusqu'à une véritable découverte du continent nord-américain (Morison, $1971: 210-13$ ).

${ }^{3}$ Beaucoup croient à origine portugaise du célèbre Dighton Rock au Massachusetts et depuis les études d'E. Delabarre la théorie s'est bien incrustée dans plusieurs milieux que cette inscription en pierre témoigne de la présence de Gaspar Corte-Real à l'imitation d'autres inscriptions pierreuses qu'ont laissés les navigateurs portugais pour indiquer leurs découvertes sur la côte d'Afrique. N'empêche que les traces multiples et indéchiffrables du Dighton Rock semblent bien résister à toute interprétation définitive, mais la plupart de chercheurs contemporains s'accordent sur l'hypothèse d'une origine amérindienne (Delabarre, 1928 ; Lopes, 1991 : 7-22, Morison, 1971, 244-47). N'ayant pas encore eu l'occasion d'examiner cette inscription moi-même, je ne tire ni n'exclue aucune des interprétations lancées.
} 
terrestre du cartographe Collard Blaeus conservé au Museu de Marinha à Lisbonne), on continue à appeler ces endroits: Terre du Lavrador, Terre de Corte-Real, et surtout Bacalhau, nom auquel je reviendrai.

L'incursion française officielle débute assez tardivement avec les voyages de Giovanni Verrazzano, au service du roi François I ${ }^{\mathrm{er}}$, en 1524 (un bon quart de siècle après Caboto et les Corte-Real). Verrazzano a rebaptisé les terres en question: Francesca (d'après le roi) et Arcadie (d'après son roman favori de Sannazaro). Dans un deuxième voyage en 1529, Verrazzano a disparu comme, avant lui, les Corte-Real d'une manière dont l'ironie révèle sans doute l'ambition du roi français de répéter la découverte portugaise par un périple presque identique mais voué à reprendre ces mêmes terres pour la France. Je rappelle entre parenthèses que François $\mathrm{I}^{\mathrm{er}}$ s'appuyait sur une interprétation créative du Traité de Tordesillas, selon laquelle les droits de possession espagnoles ou portugaises sur les terres découvertes ne pouvaient s'assurer qu'à condition que ces nations s'établissent de manière continue, à savoir une colonisation propre et non seulement une simple rencontre (Julien 1948). De là s'ensuit les voyages de Jacques Cartier dès 1534 et plus tard, du chevalier de Roberval, afin d'établir une présence française au Canada, présence que personne n'arrive à contester pour plus que deux siècles. Quel était le vrai enjeu de cette pulsion colonisatrice, qui à vrai dire, exception faite des villes passablement petites à l'époque de Québec et de Montréal, n'arrivait jamais à l'envergure d'un véritable peuplement, telles les colonies britanniques au sud du Canada ou le vaste empire espagnol depuis le Mexique jusqu'à la Terre des Feux?

La réponse reste, à mon avis, avec le sens du nom le plus courant et plus répandu pour cette région, à savoir, le Bacalhau, où le "pays de la morue". La vraie richesse du Canada, à part quelques fourrures (la mode des castors au XVII ${ }^{\mathrm{e}}$ siècle), n'était pas dans les terres âpres et neigeuses mais dans les mers à l'entour, et surtout les grands bancs, qui attirèrent très tôt les pêcheurs normands et bretons, mais aussi anglais et irlandais, basques et portugais. Selon Raimondo de Soucino, qui écrit après le voyage de Caboto en 1497, l'abondance des morues en était telle qu'ils "se prennent non seulement avec le filet mais avec un panier que l'on n'a qu'à plonger dans la mer pour le retirer plein de poissons" (La Morandière, 1952, I: 216). De là, les histoires qui persistent dans ces milieux de maints voyages très précoces dans la région de Bacalhau, et c'est le nom en portugais qui se trouve inscrit sur toutes les cartes de l'époque (et aussi c'est ce même nom traduit cette fois en anglais qui nous donne le célèbre "Cape Cod" au Massachusetts). De là aussi, ces récits mythiques qui persistent malgré leur manque de fondation probante d'un Jean Cousin de Dieppe, ou d'un João Vaz de Corte Real, père de Gaspard et Miguel, qui aurait découvert la Terre-Neuve en plein $X^{\mathrm{e}}$ siècle et bien avant Colomb, et pour laquelle découverte on lui aurait décerné le tire de governador de l'île de Terceira (Julien, 1981 : 6, Lopes, $1991: 11$ ). Nonobstant la documentation très peu certaine ce ces histoires, il n'y aurait rien de 
particulièrement impossible pour que tel ou tel vaisseau de pêche venu des Açores ou même de la Normandie ait pu se rendre sans grande fanfare dans les parages des grands bancs après s'être dispersé de plus en plus loin dans les mers de l'Atlantique. On rappelle ce document, très disputé d'ailleurs, de 1514, de l'Abbaye de Beauport en Keritry en Bretagne qui demande la dîme aux "patrons pêcheurs" pour des poissons pris il y a "deux, troys, quatre, cinq, dix, vingt, trente, quarante, cincquante, sexante ans et dedans" (Julien, 1981 : 8, La Morandière, 1952, I : 225-26). D'où venaient la prodigieuse richesse de ces "patrons pêcheurs" et l'énorme marché central à Rouen et l'organisation très méthodique pour le commerce des morues depuis l'aube $d u X V l^{e}$ siècle, sinon d'une expansion soudaine et imprévue dans le commerce et l'industrie de la pêche en général et de la morue en particulier? (Julien, 1981 : 8, La Morandière, 1952, I : 222-29).

Qu'est-ce qui est arrivé alors de cette concurrence internationale où plusieurs nations s'intéressaient vivement à s'y établir et à s'y maintenir? L'avènement de la réforme peu après rendait le commerce du poisson plus important évidemment pour les pays catholiques que pour les protestants. En même temps, la hausse des prix en lbérie au $\mathrm{XVI}^{\mathrm{e}}$ siècle rendaient de moins en moins profitables les affaires de pêche pour les Basques et les Portugais, qui continuaient tout de même pendant très longtemps d'envoyer des vaisseaux dans les grands bancs mais qui se trouvaient progressivement de moins en moins nombreux (Innis). Etant donnée cette double pression, rien d'étonnant si c'est la France qui a gagné dans ce marché et pour qui l'accès aux environs des grands bancs de la Terre Neuve devient indispensable. Pour lors, on comprend mieux pourquoi au moment de négocier la paix en 1763 avec l'Angleterre à la fin de la Guerre de Sept Ans, la France accepte bel et bien la perte de l'énorme colonie du Canada proprement dite tout en insistant sans compromis que la France garde ses accès privilégiés aux grands bancs et aux petites îles de Saint-Pierre et Miquelon, qui y furent d'importantes escales pour les pêcheurs français. De fait, et malgré les vicissitudes des derniers siècles, ces deux îles restent de nos jours une collectivité territoriale de la France (COM), et la question des droits de pêche reste un point délicat maintenant qu'après un demi millénaire de pillage, les grands bancs se trouvent presque vidés de leur stock légendaire en abondance inouïe de morues. L'idée qu'on pouvait les pêcher "avec un panier" nous fait remonter ainsi à une époque très bien révolue.

On ne peut qu'esquisser ici de manière le plus sommaire que possible la grande rivalité entre la France et le Portugal au $X V I^{e}$ siècle en ce qui concerne le Brésil. II faut quand même mentionner le voyage de Paulmier de Gonneville entrepris trois ans après la grande découverte de Pedro Alvares Cabral. L'ambition de Gonneville en était pourtant bien plus grande, car ce qui l'attirait c'étaient "les belles richesses d'épiceries et autres raretés" qu'il observait à Lisbonne comme fruits de l'épique voyage de Vasco de Gama. Avec l'armateur Jean l'Anglois (qui sera finalement mis à mort par les autorités portugaises en tant que "pirate"), il organise ce qu'il appelle ouvertement un "complot" pour concourir dans le marché 
de l'Inde à l'aide d'un vaisseau et deux Portugais qu'il dit prendre "à gros gages" pour lui servir de pilotes (Julien, 1981 : 40). Ce qui arrive ensuite à Gonneville répète exactement ce qui est arrivé à Cabral trois ans plus tôt, à savoir de s'y trouver dans des vents féroces (tels l'Adamastor de Camões) qui le repoussent violemment du Cap de la Bonne Espérance et vers les côtes du Brésil. Après quelques aventures dans ce pays, Gonneville se redirige pour la France et se voit sur le point d'y arriver quand surgit un pirate anglais dont l'attaque précipite le naufrage.

Au milieu du siècle, l'Amiral de la marine française, Gaspar de Coligny, tâche d'organiser l'établissement d'une colonie dans la Baie de Guanabara, site de la ville actuelle de Rio de Janeiro. L'idée de Coligny, à l'aube des guerres de religion en France, fut d'établir des colonies dans le nouveau monde qui puissent servir d'asile pour les huguenots persécutés. Cette colonie dura presque cinq ans, de 1555 à 1560, quand l'armada du governador geral portugais, Mem de Sá, arrive à enlever le fort français et à chasser définitivement les Français du Brésil, que ceux-ci auraient voulu nommer la France Antarctique. De ces événements, il nous reste trois grands chef d'œuvres, qui mériteraient chacun une très longue discussion: Les Singularitez de la France Antarctique, du moine franciscain et cosmographe du roi de France, André Thevet, publié à Paris en 1557 ; L'histoire d'un voyage faict en la terre du Brésil, du calviniste dévot, Jean de Léry, publié à Genève en 1578 ; et De rebus gestis Mendi de Saa Praesidis in Brasilia, une épopée latine anonyme (attribué à José d'Anchieta) inspirée de l'Enéide et publiée en 1563 à Coïmbra, et qui raconte la victoire de Mem de Sá, non pas sur des Français mais sur des "Luthériens". Quant aux deux premiers textes, la bibliographie critique est immense. Thevet et de Léry, tout en poursuivant les guerres de religion à travers leur polémique féroce et parfois farouche de ce qui s'est réellement passé au Fort de Coligny, ont tous les deux contribué de manière profonde au développement du mythe du bon sauvage, qu'ils dépeignent chacun sous le portrait du cannibale Tupinamba (Lestringant). Ce qui reste à analyser ce sera pourtant et en même temps le portrait défavorable qu'ils font des Portugais, et pour lors le rôle complémentaire de ces deux grands textes français dans la rivalité impériale avec le Portugal. Chez les deux auteurs français, il existe à la fois une peur et une arrogance envers les Portugais qui effectivement les situent dans un rapport triangulaire entre le Français et le cannibale sauvage, ce qui sera l'objet d'une étude ultérieure. Qu'il s'agit de décrire les Portugais victimes des violences anthropophagiques des Tupis ${ }^{4}$, ou bien des pirateries sadiques que leur font les matelots français qui mènent nos bons dévots en voyage (de Léry, 1578 : Ch II), le Portugais s'avère être à la fois le rival dangereux et le double qui provoque la pitié, ambiguïté qui reflète assez bien la politique ambiguë qui à la fin du $\mathrm{XVI} \mathrm{I}^{\mathrm{e}}$ siècle, et puis ironiquement encore au début du $\mathrm{XIX}^{\mathrm{e}}$ siècle dans le sillage sanglant de Napoléon, ne

\footnotetext{
${ }^{4}$ A noter l'inversion de ce rapport beaucoup plus tard en 1971 dans le film brésilien de Nelson Pereira dos Santos, " Como Era Gostoso o Meu Francês ", où c'est un Français qui se trouve l'objet de la gourmandise tupie.
} 
finit que par diminuer ces deux pays à la grande avantage d'autres rivaux (hispanique et anglais, notamment). Peut-être est-ce aussi pourquoi il en résulte vivement le dialogue culturel et littéraire entre ces deux pays, dialogue auquel nous participons encore aujourd'hui dans le colloque que voici. 


\section{Bibliographie}

ANONYME (1906). Documentos relativos a Mem de Sá, Governador Geral do Brasil. Rio de Janeiro : Officina Typographica da Bibliotheca Nacional.

[ANCHIETA, José de] (1563). De rebus gestis Mendi de Saa Praesidis in Brasilia, Rio de Janeiro: 1958: Arquivo Nacional.

Bontier, Pierre et Le VerRier, Jean (1490). Le Canarien : Codex Montruffet, Bibliothèque municipale de Rouen.

CAMÕES, Luiz Vaz de (1572). Os Lusíadas. Lisboa: Antonio Gonsalvez.

DE LERY, Jean (1578). L'histoire d'un voyage faict en la terre du Brésil. Génève : Antoine Chuppin.

DelabarRe, Edmund Burke (1928). Dighton Rock, A Study of the Written Rocks of New England. New York : W. Neale.

FernandeZ Duro, Cesáreo (1900). "Traslado de los capítulos del tratado de paces entre las coronas de Castilla y de Portugal firmado en Toledo á 16 de Marzo de 1480, relátivos á la posesión y pertenencia de Guinea, costas, mares é islas de África ", In: Boletin de la Real Academia de la Historia, tomo 36, pp. 325-29.

INNIS, Harold (1940). The Cod Fisheries: The History of an International Economy. New Haven : Yale University Press.

JULIEN, Charles-André (1948). Les voyages de découverte et les premiers établissements (XVe-XVle siècles). Paris : PUF.

JULIEN, Charles-André (éd.) (1981). Voyages au Canada : Jacques Cartier, avec les relations de voyages de Gonneville, Verrazano et Roberval. Paris : Maspero.

LA MORANDIERE, Charles de (1952). Histoire de la pêche française de la morue dans l'Amérique septentrionale. Paris : G-P Maisonneuve et Larose.

LeStRINGANT, Frank (1992). Le Huguenot et le sauvage. Paris : Klincksieck

LOPES, Francisco Fernandes (1991). The Brothers Corte Real. Lisboa: Instituto de Investigação Científica e Tropical, Edições Culturais da Marinha.

MORISON, Samuel Eliot (1971). The European Discovery of America I: The Northern Voyages. Oxford : Oxford University Press.

PeReIRA, José António Rodrigues (2010). Marinha Portuguesa: nove séculos de história. Lisboa : Comissão Cultural de Marinha.

Russel-Wood, A. J. R. (1992). The Portuguese Sea-Empire, 1415-1808. Baltimore : Johns Hopkins University Press.

SANNAZARO, Jacopo (1502). Arcadia. Venezia: Per Bartholomeo ditto I'Imperadore.

SANTOS, Nelson Pereira dos (1971). Como Era Gostoso o Meu Francês. Brésil : long métrage de 84 minutes.

THEVET, André (1557). Les singularitez de la France Antarctique. Paris : Maurice de la Porte. 